\title{
Etiopatogenia e Tratamento da Contractura Capsular Mamária
}

\author{
Etiopathogenesis and Treatment of Breast Capsular Contracture
}

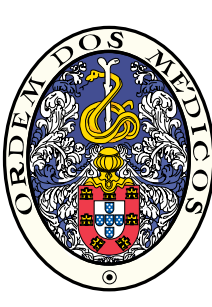

\author{
Luís PEREIRA LEITE ${ }^{1}$, Inês CORREIA SÁ², Marisa MARQUES²
}

Acta Med Port 2013 Nov-Dec;26(6):737-745

RESUMO

Introdução: A contractura capsular é a complicação crónica mais frequente da mamoplastia de aumento com próteses mamárias e a principal causa de insatisfação da doente e do cirurgião plástico. A cápsula mamária consiste num tecido fibroso que circunda a prótese e que pode contrair, alterando a forma e a consistência da mama. No estádio mais avançado é acompanhada de deformidade acentuada, rigidez e dor, tendo indicação para tratamento cirúrgico.

Material e Métodos: Foram revistos todos os artigos indexados na PubMed através da pesquisa 'capsular contracture' (2000 - Janeiro 2012), dos quais foram inseridos os artigos de maior interesse em termos de etiologia, profilaxia e tratamento. Artigos referenciados em publicações relevantes foram também analisados.

Resultados: Tudo indica que a sua etiologia é multifactorial; a etiopatogenia da contractura capsular mamária continua a ser alvo de múltipla investigação pré-clínica. Vários são os estudos realizados de forma a prevenir a ocorrência de contractura capsular e, embora os resultados sejam promissores, pouco está definido em termos da sua aplicação na prática clínica. Relativamente ao tratamento a capsulectomia/capsulotomia continua a ser o gold-standard, no entanto o futuro poderá passar por técnicas não invasivas, pelo menos em estadios mais leves da doença.

Conclusão: Apesar das técnicas cirúrgicas e a qualidade das próteses mamárias terem vindo a melhorar drasticamente nos últimos anos, a contractura capsular mamária mantém-se uma complicação real, com incidência elevada e que continua a afectar milhares de mulheres no mundo.

Palavras-chave: Contratura Capsular em Implantes; Mamoplastia; Próteses Mamárias.

\section{ABSTRACT}

Introduction: Capsular contracture is a chronic and the most frequent complication of augmentation mammoplasty with breast implants and the main cause of patient's and surgeon's dissatisfaction. The mammary capsule consists of a fibrous tissue that surrounds the implant that may contract, changing the shape and consistency of the breast. In its advanced stage is accompanied by pronounced deformity, hardness and pain, being indicated for surgical treatment.

Material and Methods: All the articles indexed on PubMed through the search 'capsular contracture' (2000 - January 2012) were reviewed and were included the articles of greater interest in terms of etiology, prophylaxis and treatment. Articles referred in relevant publications were also examined.

Results: Everything indicates that its etiology is multifactorial; the etiopathology of breast capsular contracture continues being subject of multiple pre-clinical investigations. There are many studies performed in order to prevent the onset of capsular contracture but, although promising results, little is set for its application on clinical practice. The capsulectomy/capsulotomy continues being the goldstandard treatment although the future may undergo non invasive techniques, at least in mild stages of disease.

Conclusion: Although the surgical techniques and the quality of breast implants have been improving drastically in recent years, capsular contracture remains a real complication with great incidence and that continues affecting thousands of women all over the world. Keywords: Breast Implants; Implant Capsular Contracture; Mammaplasty.

\section{INTRODUÇÃO}

A contractura capsular (CC) é a complicação mais frequente após mamoplastia de aumento, ${ }^{1-4}$ bem como a mais importante causa de insatisfação tanto para a doente como para o cirurgião plástico. Surgiu praticamente aquando da introdução de próteses de silicone por Cronin e Gerow, em $1963^{5}$ e, apesar de quase cinco décadas decorridas, a sua prevalência e incidência continuam elevadas, afectando milhares de mulheres no mundo. Estudos recentes apontam para valores de incidência de CC na ordem dos $16 \%$. $^{1,2}$ Em Portugal, num estudo realizado recentemente no Hospital de São João, a incidência de CC atingiu os $23,1 \%$, apresentando o grupo estético uma incidência menor com- parativamente ao grupo reconstrutivo (11\% vs $37,4 \%){ }^{6}$

A formação da cápsula faz parte de uma resposta adaptativa universal a um corpo estranho, neste caso a prótese de silicone que, apesar de relativamente inerte, provoca esta reacção imunológica de protecção. ${ }^{7}$ Apesar de as cápsulas apresentarem geralmente um carácter benéfico, isolando o material exógeno e mantendo a prótese no local de implantação, ${ }^{8}$ os problemas surgem quando se tornam patologicamente activas passando por um processo de 'fibrose constritiva' que deforma o conteúdo e prejudica o êxito estético, ${ }^{9}$ levando à rigidez, tensão e deformação da mama e possibilidade de ocorrência de dor. ${ }^{8}$

1. Departamento de Cirurgia. Faculdade de Medicina. Universidade do Porto. Porto. Portugal.

2. Serviço de Cirurgia Plástica, Reconstrutiva e Estética. Departamento de Cirurgia - FMUP. Hospital de São João. Porto. Portugal.

Recebido: 05 de Fevereiro de 2012 - Aceite: 15 de Julho de 2013 | Copyright $\odot$ Ordem dos Médicos 2013 
A classificação geralmente utilizada baseia-se na classificação de Baker, ${ }^{10}$ que define os estádios de CC baseado na apresentação clínica das doentes (Tabela 1). O grau II é o primeiro estádio de $\mathrm{CC}$ e a interpretação clínica deste é altamente dependente da opinião individual dos cirurgiões. Apesar do impacto clínico de uma CC de grau II ser relevante, a maioria dos estudos, tanto prospectivos como retrospectivos, não inclui este estádio na análise da CC..$^{11-14}$ Marques et $a^{6}$ sugerem que o grau II deverá ser sempre incluído na análise da CC, bem como um follow-up superior a 42 meses. Uma outra classificação, introduzida por Wilflingseder et al ${ }^{15}$ baseia-se nos achados histológicos da cápsula (Tabela 2). Estudos recentes confirmam uma correlação positiva entre a classificação clínica de Baker e a classificação histológica de Wilflingseder. ${ }^{16,17}$

Com este trabalho o autor pretende realizar uma revisão acerca da CC, focando os últimos avanços em termos de etiologia, profilaxia e tratamento, ciente no interesse e utilidade deste artigo numa época em que a mamoplastia de aumento por razões estéticas tem vindo a aumentar e a reconstrução mamária assume-se fundamental como complemento da mastectomia.

\section{MATERIAL E MÉTODOS}

Foi realizada uma pesquisa sistemática da literatura existente, desde Janeiro de 2000 até Maio de 2012, através da base de dados bibliográfica biomédica. Utilizou-se como palavra-chave o termo 'Capsular Contracture'; foram obtidos 506 resultados sendo a selecção dos mesmos feita tendo em conta a sua adequação aos objectivos desta revisão. A informação foi dividida, genericamente, em três partes: Etiopatogenia; Profilaxia; Tratamento. Foram ainda incluídas referências a publicações relevantes datadas antes de 2000.

\section{Etiopatogenia}

A etiologia da CC não está bem definida, sendo que a maioria dos autores apontam para uma etiologia multifactorial, que conjuga a hipótese infecciosa com a hipótese inflamatória.

\section{Hipótese infecciosa}

A hipótese infecciosa, baseada numa relação causal entre a infecção subclínica e o desenvolvimento de CC há muito que está proposta. ${ }^{18}$ As potenciais fontes de contaminação incluem: prótese contaminada; soro fisiológico injectável contaminado; ambiente cirúrgico não estéril; flora cutânea ou ductos mamários; e invasão bacteriana com origem noutros locais. ${ }^{19} \mathrm{O}$ próprio tecido mamário pode ser fonte de infecção, sendo os locais de maior concentração bacteriana as áreas cujo tecido ductal é maior, como sejam a região peri-areolar ou infra-mamária. ${ }^{20}$

Vários estudos prospectivos apresentam resultados estatisticamente significativos entre a existência de infecção e a presença de CC grave. ${ }^{11-24}$ Num estudo pré-clínico comprovou-se que a formação de biofilme aumenta em quatro vezes o risco de se desenvolver $\mathrm{CC}$, demonstrando-se uma relação causal. ${ }^{25}$ Schreml et al ${ }^{22}$ defendem que o estímulo bacteriano promove a inflamação e fibrose e consequentemente a CC ao constatarem que nenhuma doente com grau I ou II de Baker evidenciou colonização capsular, em comparação com taxas de colonização na ordem dos

Tabela 1- Classificação de Baker
Grau
Descrição

I Mama de aspecto natural - mamoplastia de aumento não perceptível.

II Contractura leve - perceptível pelo cirurgião; doente assintomática.

III Contractura moderada - doente sente alguma firmeza.

IV Contractura grave - evidente à observação.

Tabela 2 - Classificação de Wilflingseder

\begin{tabular}{cl}
\hline Grau & Descrição \\
\hline II $\quad$ Cápsula fina e não contraída. & 'Fibrose constritiva' sem células gigantes. \\
III $\quad$ 'Fibrose constritiva' com células gigantes presentes. \\
IV $\quad$ Células inflamatórias, granulomas de corpo estranho, neovascularização, possibilidade de ocorrência de neuromas.
\end{tabular}


$66,7 \%$ em contracturas grau III ou IV de Baker. Os microrganismos mais comummente encontrados são várias espécies de Staphylococcus coagulase-negativos e o Propionibacterium acnes, sendo o $\mathrm{S}$. epidermidis o mais implicado na infecção capsular. ${ }^{20,21,23}$

\section{Hipótese inflamatória}

Tem-se sugerido que a inflamação crónica desempenha um papel fundamental na etiopatogenia da CC e inclusivamente já foram identificados vários mediadores inflamatórios envolvidos como o TGF- $\beta$, TNF- $\alpha$, entre outros. A presença de grande número de macrófagos contendo silicone associada ao aumento da inflamação local no tecido capsular, ${ }^{17}$ bem como a presença de células gigantes multinucleares e metaplasia tipo sinovial na camada mais interna das cápsulas ${ }^{26}$ sugere o mecanismo inflamatório como factor-chave no desenvolvimento de CC. Também a presença de miofibroblastos no tecido peri-protésico, já observado desde $1981,{ }^{27}$ favorece esta teoria. Recentemente, Hwang e $\mathrm{a}^{28}$ demonstraram o aparecimento dos miofibroblastos durante a fase activa da contracção cicatricial, diminuindo posteriormente aquando da sua maturação.

Em 2007, um estudo preliminar demonstrou a expressão de elevados níveis de mRNA da citocina pró inflamatória TNF-a e do receptor de leucotrienos tipo II em cápsulas severamente contraídas. ${ }^{29}$ Posteriormente, confirmou-se o aumento significativo na expressão dos receptores de leucotrienos em doentes com CC severa, providenciando parcialmente uma base biomolecular que justifica o uso de anti-leucotrienos no tratamento desta doença. ${ }^{30}$

Ulrich et $\mathrm{al}^{31}$ demonstraram um aumento das concentrações séricas da metaloproteinase MMP-2 e dos inibidores das metaloproteinases TIMP-1 e TIMP-2 nas doentes com $\mathrm{CC}$, bem como uma diminuição do ratio MMP/TIMP, valores correlacionados com o estádio de Baker previamente estabelecido. Num outro estudo observaram resultados semelhantes, evidenciando aumentos mais marcados na expressão dos TIMP's em próteses lisas, comparativamente às próteses texturadas. ${ }^{32}$ Estes resultados sugerem que as concentrações elevadas dos TIMP's poderão estar envolvidas na patogénese da CC, bem como poderão explicar as maiores taxas de CC observadas em próteses lisas. No futuro, um tratamento não invasivo que diminua os TIMP's e aumente a actividade das MMP's poderá ser útil na profilaxia desta grave complicação.

O envolvimento da citocina TNF- $\alpha$ como possível mediador inflamatório fulcral envolvido na patogénese da CC foi recentemente verificado, observando-se um aumento da sua expressão associado ao aumento do grau de severidade de CC, estando presente em fibroblastos, macrófagos e tecido extracelular próximo da prótese..$^{33}$

\section{O papel da radioterapia}

O efeito nocivo da radioterapia como indutor da CC há muito que está descrito, ${ }^{34}$ sendo vários os estudos que correlacionam positivamente tal facto; ${ }^{34-46}$ inclusivamente uma investigação recente utiliza a radiação direta como forma de indução de CC num modelo animal. ${ }^{36} \mathrm{~A}$ incidência de CC no grupo de mulheres que recebe radioterapia (RT) é largamente superior à do grupo não irradiado (NRT), como sugerem os resultados obtidos recentemente: RT-21,6\% vs NRT-3,3\%. ${ }^{41}$ As imediações das zonas previamente irradiadas são os locais mais frequentemente afectados. ${ }^{43}$ Katzel et $\mathrm{al}^{35}$ demonstraram que parte deste fenómeno de lesão radioactiva deve-se ao processo de activação da cascata das citocinas, sendo a activação do TGF- $\beta$ o principal mecanismo envolvido. Nesse mesmo estudo verificou-se que a inibição da sinalização do TGF- $\beta$ levou a uma redução na incidência de $\mathrm{CC}$ relacionada com a radiação, abrindo perspectivas futuras para utilização de terapias específicas anti-Smad3/TGF- $\beta$.

\section{Profilaxia}

Vários estudos têm sido publicados sobre a influência de determinados fármacos na prevenção da CC, assim como têm sido desenvolvidas novas técnicas cirúrgicas e novos tipos de próteses com o intuito de diminuir a incidência desta tão debilitante complicação.

\section{Factores de risco}

Num estudo retrospectivo recente verificou-se que a marca da prótese, a presença de infecção subjacente, a ocorrência de hematoma tardio e a gravidez aumentam significativamente o risco de CC. ${ }^{47} \mathrm{O}$ índice de massa corporal, o hábito tabágico e o consumo de álcool não obtiveram associação significativa com o desenvolvimento de CC, resultados semelhantes aos obtidos noutros estudos. ${ }^{6,12} \mathrm{~A}$ terapêutica hormonal também não está implicada no desenvolvimento de $C C,{ }^{6,12,38}$ não havendo relação entre $C C$ e menopausa ou estado estrogénico.

\section{Human Acellular Dermal Matrices - Alloderm ${ }^{\circledR}$}

A utilização de matrizes dérmicas não celulares em mamoplastias de aumento tem sido associada a uma diminuição da incidência de CC..$^{48-51}$ Orenstein et al ${ }^{52}$ demonstraram, in vitro, que o Alloderm ${ }^{\circledR}$ diminuía significativamente as concentrações dos mediadores inflamatórios IL-1ß, IL-6, IL-8 e VEGF, quando colocado em contacto com células mononucleares de sangue periférico humano. Num estudo pré-clínico a utilização de Alloderm $^{\circledR}$ demonstrou uma inibição completa da formação capsular, diminuindo significativamente a formação de miofibroblastos, provavelmente devido à sua acção de barreira e prevenção da iniciação do processo de 'reacção de corpo estranho'. ${ }^{53}$ De igual forma, estudos efectuados através de biópsias e avaliações histológicas de cápsulas mamárias humanas parecem confirmar a diminuição dos vários parâmetros de fibrose capsular, quando são utilizadas matrizes dérmicas não celulares. ${ }^{54}$ No entanto, e apesar das inúmeras vantagens que parecem advir da sua utilização, estas encontram-se associadas a um preço elevado, por vezes incomportável para vários centros médicos. 


\section{Materiais de preenchimento}

Desde a introdução das próteses de silicone, estas sofreram várias alterações. Com o objectivo de reduzir as taxas de complicações, nomeadamente CC, bem como melhorar o aspeto estético, surgiram no mercado cinco gerações de próteses de silicone, que culminam nas próteses de gel coesivo introduzidas em 1993. Recentemente foi realizada uma meta-análise (a primeira e única até ao momento) para avaliar o efeito do tipo de material utilizado no preenchimento de próteses mamárias e as taxas de CC..$^{55}$ Os resultados indicam uma maior taxa de CC em doentes que receberam próteses de silicone, quando comparadas com as que receberam próteses de soro fisiológico. No entanto, estes resultados não poderão ser extrapolados para a população pois não incluem a avaliação da mais recente geração de próteses de silicone (mais utilizadas actualmente).

\section{Superfície}

As propriedades físicas recentemente desenvolvidas aplicadas à construção das superfícies protésicas (o processo de texturização) também contribuíram para diminuição da CC. Tem sido sugerido que a formação de uma cápsula contínua com fibras de colagénio dispostas paralela e circularmente ao redor das próteses lisas promove o desenvolvimento de forças contrácteis concêntricas, que tracionando em conjunto desenvolvem uma contractura. ${ }^{56}$

A maioria dos estudos recentes revela que as próteses texturadas apresentam incidências significativamente menores de CC, em comparação com as próteses lisas, ${ }^{56-59}$ embora haja estudos que não evidenciam diferenças significativas. ${ }^{60}$ Considera-se que o crescimento tecidular nas próteses texturadas origina forças contrácteis multidireccionais com tendência a neutralizarem-se entre si. ${ }^{56}$ Barr et al ${ }^{61}$ utilizaram técnicas de microscopia electrónica de varrimento de alta resolução para avaliar as diferentes topografias de superfície de algumas das próteses mais utilizadas (lisas e texturadas); observaram a superfície de contacto entre a prótese e o tecido e confirmaram a capacidade das próteses texturadas contrariar a disposição paralela referida anteriormente. Concluem e incentivam à descoberta de novos materiais, baseados em nanotecnologia e bioengenharia, que sejam biologicamente mais compatíveis e menos dependentes da aleatoriedade observada com as próteses actuais.

Próteses revestidas com espuma de poliuretano têm sido utilizadas desde a sua introdução em 1970 por Ashley et al $^{62}$ Desde cedo apresentaram bons resultados, aliados a uma baixa taxa de complicações, nomeadamente baixa incidência de $C C,{ }^{63-65}$ que atinge valores tão baixos como $0,4 \% .^{13} \mathrm{~A}$ possibilidade da absorção do poliuretano e sua consequente degradação em 2,4-toluenediamina (2,4-TDA) foi considerada carcinogénica. ${ }^{66,67}$ Esta associação foi desmentida pela FDA em $1995^{68}$ que considerou estas próteses seguras ao demonstrar concentrações de 2,4-TDA na urina extremamente baixas e níveis inexistentes de TDA livre no plasma em doentes com próteses de poliuretano. Handel et $a^{69}$ concluem que o perfil de segurança deste tipo de próteses é idêntico ao observado noutros dispositivos de silicone, enquanto Pena-Salcedo et $\mathrm{al}^{13}$ afirmam que estas próteses são a melhor escolha para utilização em mamoplastias de aumento. Apesar disso, o metabolismo do poliuretano continua por esclarecer, razão pela qual a maioria dos cirurgiões plásticos não aderiu à sua utilização como primeira escolha.

\section{Antibioterapia, corticoterapia e outros:}

Num estudo em que as doentes foram submetidas a profilaxia antibiótica pré, intra e pós-operatoriamente Carlesimo et al $^{70}$ obtiveram apenas $10 \%$ de doentes com Baker grau II, não observando qualquer caso de Baker grau III ou IV. Os autores acreditam que o processo infeccioso é a principal causa de CC e, como tal, prevenindo a sua activação através da terapêutica antibiótica e utilizando uma abordagem axilar, que permite menor risco de contaminação, é possível a prevenção da CC. Adams et al ${ }^{11}$ consideram a profilaxia antibiótica de extrema importância, propondo a lavagem do local de implantação com antibioterapia tripla, intra-operatoriamente; este procedimento mostrou-se extremamente eficaz e custo-efectivo na prevenção da CC. A impregnação das próteses com agentes antimicrobianos também é proposta, evidenciando uma diminuição significativa da colonização bacteriana e da CC. ${ }^{71} \mathrm{Um}$ estudo recente defende que uma única dose de antibiótico intravenoso é adequada para profilaxia da $\mathrm{CC}$, e a cobertura antibiótica adicional não reduz o risco de infecções superficiais ou peri-protésicas. ${ }^{72}$

A utilização de glicocorticóides na prevenção (ou tratamento) da CC apresenta grande potencial, no entanto as doses necessárias para obtenção de eficácia terapêutica resultam em efeitos laterais sistémicos significativos, impedindo o seu uso. ${ }^{73-75} \mathrm{~A}$ utilização de fármacos associados a lipossomas permite uma libertação mais eficiente e contínua dos compostos terapêuticos; desta forma a utilização de prednisolona lipossómica permitiu uma redução em cerca de dez vezes a sua dosagem habitual, diminuindo assim os efeitos adversos sistémicos. ${ }^{76}$ Moreira et al ${ }^{77}$ testaram o efeito da administração local de uma única dose de prednisolona fosfato-lipossómica (PPL) na formação capsular e observaram uma diminuição significativa da espessura da cápsula e da densidade de colagénio, bem como uma redução significativa na contagem de miofibroblastos. Estes resultados, aliados a um efeito sistémico diminuto, fazem da PPL uma importante ferramenta profiláctica.

Em 2007, Gancedo et al ${ }^{78}$ investigaram a acção do imunomodulador pirfenidona sobre as cápsulas mamárias em camundongos, obtendo resultados promissores. Verificaram no grupo experimental uma diminuição da espessura da cápsula, da proliferação de células tipo fibroblasto e do recrutamento de células inflamatórias, bem como uma diminuição generalizada dos mediadores inflamatórios.

Um estudo pré-clínico demonstrou uma redução significativa do processo inflamatório da cápsula através da administração de inibidores da enzima de conversão da 
angiotensina (enalapril); observou-se uma diminuição da expressão de TGF-ß1 e dos anticorpos anti-ED1 e anti-colagénio tipo III. ${ }^{79}$ Estes resultados poderão providenciar uma base terapêutica económica e segura na diminuição da incidência de CC.

A aplicação de bloqueadores dos canais de cálcio também tem sido investigada devido ao mecanismo de acção do cálcio, que actua como segundo mensageiro na produção e libertação de colagénio e fibronectina pelos fibroblastos. ${ }^{80}$ Benlier et al ${ }^{81}$ obtiveram uma diminuição significativa da espessura da cápsula quando estas eram instiladas previamente com $5 \mathrm{mg}$ de verapamil, em camundongos.

Marisa Marques et al ${ }^{82,83}$ demonstraram em dois estudos pré-clínicos a vantagem da utilização das colas de fibrina comerciais Tisseel ${ }^{\circledR} /$ Tissucol $^{\circledR}$ na profilaxia da CC ao comprovar pressões intra-capsulares significativamente menores no grupo intervencionado. A cola de fibrina é estudada, há décadas, pelo seu uso cirúrgico como agente hemostático e promotor da cicatrização, sendo rotineiramente utilizada em diversos processos cirúrgicos - a salientar: anastomoses gastrointestinais, neurorrafias, consolidação de enxertos, neurocirurgia e oftalmologia. ${ }^{84-92}$ Estas colas, com um perfil de segurança relativamente bem documentado, reduzem a formação de matriz extracelular e de TGF- $\beta,{ }^{93}$ apresentando-se como um agente profiláctico atractivo capaz de modificar a formação da cápsula e, assim, atenuar o subsequente desenvolvimento de CC.

Também recentemente têm-se tentado sintetizar novos fármacos capazes de inibir a actividade do TGF- $\beta$. Ruiz-de-Erenchun et al ${ }^{94}$ demonstraram uma redução na formação de fibrose peri-protésica, num estudo pré-clínico com um peptídeo inibidor do TGF- $\beta$. Em 2011 Diao et al ${ }^{95}$ publicaram um estudo pré-clínico no qual obtêm uma prevenção eficaz da formação capsular através da utilização de um inibidor da cínase do receptor de TGF- $\beta$, de libertação controlada por um hidrogel termo sensível desenvolvido para o efeito. O grupo experimental, comparativamente com o grupo controle, apresentou uma redução significativa: da espessura da cápsula; da angiogénese; do infiltrado de células inflamatórias; de fibroblastos; e da expressão de mRNA de colagénio tipo III e TGF- $\beta 1$.

A utilização de oxigénio hiperbárico pós radioterapia demonstrou eficácia, ${ }^{96}$ assim como a aplicação precoce e repetida de ultrassons externos pós-implantação ${ }^{97}$ e a electroestimulação ao terceiro dia pós-operatório com intensidades de $600 \mathrm{mV} .^{98} \mathrm{O}$ agente antioxidante quimioterápico mesna também demonstrou eficácia na diminuição da espessura da cápsula, apresentando-se como um promissor adjuvante, ${ }^{99}$ tal como o líquido amniótico humano. ${ }^{100} \mathrm{~A}$ compressão mamária é aconselhável; baseados em fórmulas matemáticas, Camirand et al ${ }^{101}$ comprovaram a sua eficácia na prevenção da CC.

\section{Tratamentro}

O tratamento da CC baseia-se actualmente na aplicação de procedimentos cirúrgicos, como sejam a capsulotomia ou a capsulectomia (parcial ou total), ou procedimentos médicos, os quais não são genericamente aceites, estando a maioria ainda em fase de avaliação clínica. Os objectivos primordiais são a resolução da contractura e a prevenção da sua recorrência.

\section{Procedimentos cirúrgicos}

Ambos os procedimentos cirúrgicos (capsulotomia ou capsulectomia) envolvem a substituição da prótese e eventualmente a sua introdução num novo espaço tecidular. A capsulectomia total cria um novo espaço para a recolocação da prótese e demonstrou menores taxas de recorrência comparativamente à capsulectomia parcial. ${ }^{102}$ Outras técnicas comummente utilizadas consistem no reposicionamento da prótese retroglandularmente se previamente retropeitoral ou vice-versa, bem como, mais recentemente, o reposicionamento no chamado dual-plane, no qual os dois terços superiores da prótese são colocados numa posição retropeitoral e o terço inferior é posicionado retroglandularmente. ${ }^{103,104}$ Baran et al ${ }^{105}$ apresentaram uma técnica diferente que consistia em deixar a cápsula intacta e colocar uma nova prótese imediatamente acima ou abaixo dessa cápsula. Observaram que praticamente todo o tecido capsular se torna mole e maleável logo após a remoção da prótese não actuando, portanto, como impeditivo à colocação de uma nova prótese na proximidade. Xue et al ${ }^{106}$ também defendem a manutenção da cápsula e colocação de uma nova prótese anteriormente a esta. A abordagem descrita é axilar, apresenta-se minimamente invasiva e simples de realizar. Actualmente fica ao critério do cirurgião, com base na sua experiência e resultados, a escolha da melhor opção cirúrgica. Seria importante a realização de estudos comparativos entre as diferentes opções cirúrgicas que permitam avaliar a sua eficácia e efectividade.

\section{Inibidores dos leucotrienos}

Em 2002, Schlesinger et al ${ }^{107}$ demonstraram a diminuição significativa do grau de CC com a administração oral de zafirlukast. Desde então vários estudos procedentes justificam o seu uso na prática clínica explicando o mecanismo de acção envolvido ${ }^{29,30}$ ou demonstrando as alterações histológicas subsequentes. ${ }^{108-110}$ Vários autores publicaram também os seus resultados clínicos que apoiam, de facto, a eficácia dos inibidores dos leucotrienos no tratamento da $\mathrm{CC}$, evidenciando genericamente uma diminuição da dor e da distorção mamária associadas. ${ }^{111,112}$ Resultados semelhantes são também obtidos utilizando montelukast. ${ }^{113}$ Apesar disso, o uso de zafirlukast merece um acompanhamento cuidadoso pois a utilização de doses habituais do fármaco tem sido associada ao desenvolvimento de hepatite e insuficiência hepática numa pequena percentagem de doentes, que pode ocorrer dois a oito meses após a sua toma. ${ }^{114,115}$ Curiosamente, estudos realizados com montelukast não mostraram diferenças significativas em termos de função hepática comparativamente ao grupo placebo, ${ }^{116}$ apesar da existência de relatos de hepatites induzidas pela toma deste fármaco. ${ }^{117}$ É necessário ponderar os riscos e benefícios envolvidos na toma de inibidores dos leucotrie- 
nos no tratamento da CC.

\section{Outros fármacos}

Outros fármacos recentemente estudados para o tratamento da CC incluem a toma oral de pirfenidona, que apresenta propriedades anti-fibróticas e anti-inflamatórias, e que demonstrou eficácia na melhoria do score de Baker previamente atribuído ${ }^{118} \mathrm{e}$ a injeção percutânea de triancinolona-de-acetonido guiada por ultrassonografia, que mostrou-se eficaz no tratamento de CC de grau IV. ${ }^{119} \mathrm{~A}$ aplicação tópica, durante três semanas, de diclofenac (Fector Tissuge ${ }^{\circledR}$ ) mostrou resultados surpreendentes ao reverter os graus II e III de Baker em grau I, desde que a aplicação seja iniciada até três meses após o início da CC. ${ }^{120}$ Planas et $\mathrm{al}^{121}$ demonstraram ainda resultados excelentes com a aplicação de ultrassonografia para tratamento da CC.

\section{CONCLUSÃO}

A CC da mama é uma identidade clínica bem estabelecida que consiste na contracção do tecido envolvente à prótese, vulgo cápsula, sendo a formação desta cápsula parte de uma resposta adaptativa universal a um corpo estranho. Está associada a distorção mamária em variados graus e geralmente é acompanhada de dor, eritema ou desconforto mamários.

A etiologia da contractura permanece desconhecida, havendo controvérsia quanto ao papel da infecção, da inflamação e da autoimunidade no seu desenvolvimento; os estudos actuais apontam para uma etiologia multifactorial, na qual o TGF- $\beta$ participa e actua como importante mediador.

Em termos profilácticos, várias medidas têm sido propostas para prevenir a CC: a realização de uma hemóstase cuidadosa, o uso de drenos aspirativos, a injecção intralu-

\section{REFERÊNCIAS}

1. Kjoller K, Holmich LR, Jacobsen PH, Friis S, Fryzek J, McLaughlin JK, et al. Epidemiological investigation of local complications after cosmetic breast implant surgery in Denmark. Ann Plast Surg. 2002;48:229-337.

2. Kulmala I, McLaughlin JK, Pakkanen M, Lassila K, Holmich LR, Lipworth L, et al. Local complications after cosmetic breast implant surgery in Finland. Ann Plast Surg. 2004;53:413-9.

3. Fryzek JP, Signorello LB, Hakelius L, Lipworth L, McLaughlin JK, Blot WJ, et al. Local complications and subsequent symptom reporting among women with cosmetic breast implants. Plast Reconstr Surg. 2001;107:214-21.

4. Gabriel SE, Woods JE, O'Fallon WM, Beard CM, Kurland LT, Melton LJ 3rd. Complications leading to surgery after breast implantation. N Engl J Med. 1997;336:677-82.

5. Cronin TD, Gerow FJ. Augmentation mammoplasty: A new 'natural fee' prosthesis. In: Transactions of the 3rd International Congress of Plastic Surgery (no. 66). Amsterdam: Excerpta Medica International Congress Series; 1964. p.41.

6. Marques M, Brown SA, Oliveira I, Cordeiro MN, Morales-Helguera A, Rodrigues A, et al. Long-term follow-up of breast capsule contracture rates in cosmetic and reconstructive cases. Plast Reconstr Surg. 2010;126:769-78.

7. Vistnes LM, Bentley JW, Fogarty DC. Experimental study of tissue response to ruptured gel-filled mammary prostheses. Plast Reconstr Surg. 1977;59:31-4.

8. Puskas JE, Luebbers MT. Breast implants: the good, the bad and the ugly. Can nanotechnology improve implants? Wiley Interdiscip Rev Nanomed Nanobiotechnol. 2012;4:153-68.

9. Berry MG, Cucchiara V, Davies DM. Breast augmentation: Part minal de corticóides, antibióticos ou outros fármacos, a utilização de próteses texturadas ou de poliuretano, bem como o posicionamento das próteses em posição retropeitoral. A profilaxia antibiótica mostrou-se particularmente eficaz nessa redução, no entanto está por descobrir qual o melhor esquema terapêutico a aplicar e se pré, intra, pós-operatoriamente ou durante todo o período peri-operatório. Apesar da melhor qualidade das próteses actualmente existentes e do aprimoramento das técnicas cirúrgicas, a CC continua a ser a mais prevalente complicação das mamoplastias de aumento, estéticas ou reconstrutivas.

$\mathrm{O}$ tratamento da CC baseia-se, actualmente, em procedimentos cirúrgicos, sendo a capsulectomia total preferível à capsulotomia. Têm sido descritas técnicas cirúrgicas simples, eficazes e menos invasivas que poderão ser o futuro do tratamento cirúrgico desta complicação. Apesar da eficácia dos inibidores dos leucotrienos no tratamento da $\mathrm{CC}$, a sua possível associação com hepatite e insuficiência hepática tem diminuído a sua utilização. Importa referir que outros fármacos e procedimentos têm sido testados para o tratamento em questão, como a aplicação de ultrassonografia ou diclofenac tópico, acompanhados de bons resultados. O futuro poderá passar pela utilização destes, ou outros procedimentos, que se apresentem não invasivos e com poucos efeitos laterais.

\section{CONFLITOS DE INTERESSE}

Os autores declaram a inexistência de conflitos de interesse.

\section{FONTES DE FINANCIAMENTO}

Os autores declaram a inexistência de fontes de financiamento.

II - Adverse capsular contracture. J Plast Reconstr Aesthet Surg. 2010;63:2098-107.

10. Baker JIJW. Augmentation mammaplasty. In: Owsley JE, editor. Symposium of aesthetic surgery of the breast: Proceedings of the Symposium of the Educational Fundation of the American Society of Plastic and Reconstructive Surgeons and the American Society for Aesthetic Plast Surg., in Scottsdale, Ariz., November 23-26, 1975. St. Louis: Mosby, 1978. p. 256-63.

11. Adams WP Jr., Rios JL, Smith SJ. Enhancing patient outcomes in aesthetic and reconstructive breast surgery using triple antibiotic breast irrigation: six-year prospective clinical study. Plast Reconstr Surg. 2006;117:30-6.

12. Henriksen TF, Fryzek JP, Holmich LR, McLaughlin JK, Kjoller K, Hoyer $A P$, et al. Surgical intervention and capsular contracture after breast augmentation: a prospective study of risk factors. Ann Plast Surg. 2005;54:343-51.

13. de la Pena-Salcedo JA, Soto-Miranda MA, Lopez-Salguero JF. Back to the future: A 15-year experience with polyurethane foam-covered breast implants using the partial-subfascial technique. Aesthetic Plast Surg. 2012;36:331-8.

14. Cunningham $B$. The Mentor Core Study on silicone memorygel breast implants. Plast Reconstr Surg. 2007;120:19S-29S.

15. Mikuz G, Hoinkes G, Probst A, Wilflingseder P. Tissue reactions with silicone rubber implants (morphological, microchemical, and clinical investigations in humans and laboratory animals). In: Hastings GW, Ducheyne P, editors. Macromolecular Biomaterials. Boca Raton, Florida: CRC Press; 1983. pp.239-49.

16. Prantl L, Schreml S, Fichtner-Feigl S, Poppl N, Eisenmann-Klein M, 
Schwarze $\mathrm{H}$, et al. Clinical and morphological conditions in capsular contracture formed around silicone breast implants. Plast Reconstr Surg. 2007;120:275-84.

17. Prantl L, Schreml S, Fichtner-Feigl S, Poppl N, Roll C, Eisenmann-Klein $\mathrm{M}$, et al. Histologische und immunhistochemische Untersuchungen bei Kapselkontraktur nach glatten Brustimplantaten. Handchir Mikrochir Plast Chir. 2006;38:224-32.

18. Shah Z, Lehman JA, Jr., Tan J. Does infection play a role in breast capsular contracture? Plast Reconstr Surg. 1981;68:34-42.

19. Pittet B, Montandon D, Pittet D. Infection in breast implants. Lancet Infect Dis. 2005;5:94-106.

20. Bartsich S, Ascherman JA, Whittier S, Yao CA, Rohde C. The breast: a clean-contaminated surgical site. Aesthetic Plast Surg. 2011;31:802-6.

21. Pajkos A, Deva AK, Vickery K, Cope C, Chang L, Cossart YE. Detection of subclinical infection in significant breast implant capsules. Plast Reconstr Surg. 2003;111:1605-11.

22. Schreml S, Heine N, Eisenmann-Klein M, Prantl L. Bacterial colonization is of major relevance for high-grade capsular contracture after augmentation mammaplasty. Ann Plast Surg. 2007;59:126-30.

23. Rieger UM, Pierer G, Luscher NJ, Trampuz A. Sonication of removed breast implants for improved detection of subclinical infection. Aesthetic Plast Surg. 2009;33:404-8

24. Del Pozo JL, Tran NV, Petty PM, Johnson CH, Walsh MF, Bite U, et al. Pilot study of association of bacteria on breast implants with capsular contracture. J Clin Microbiol. 2009;47:1333-7.

25. Tamboto H, Vickery K, Deva AK. Subclinical (biofilm) infection causes capsular contracture in a porcine model following augmentation mammaplasty. Plast Reconstr Surg. 2010;126:835-42.

26. Prantl L, Angele P, Schreml S, Ulrich D, Poppl N, Eisenmann-Klein M Determination of serum fibrosis indexes in patients with capsular contracture after augmentation with smooth silicone gel implants. Plast Reconstr Surg. 2006;118:224-9.

27. Baker JL Jr, Chandler ML, LeVier RR. Occurrence and activity of myofibroblasts in human capsular tissue surrounding mammary implants. Plast Reconstr Surg. 1981;68:905-12.

28. Hwang K, Sim HB, Huan F, Kim DJ. Myofibroblasts and capsular tissue tension in breast capsular contracture. Aesthetic Plast Surg 2010;34:716-21.

29. D'Andrea F, Nicoletti GF, Grella E, Grella R, Siniscalco D, Fuccio C, et al. Modification of cysteinyl leukotriene receptor expression in capsular contracture: Preliminary results. Ann Plast Surg. 2007;58:212-4.

30. Grella E, Grella R, Siniscalco D, Fuccio C, Rossi F, De Novellis V, et al. Modification of cysteinyl leukotriene receptors expression in capsular contracture: follow-up study and definitive results. Ann Plast Surg. 2009;63:206-8

31. Ulrich D, Lichtenegger F, Eblenkamp M, Repper D, Pallua N. Matrix metalloproteinases, tissue inhibitors of metalloproteinases, aminoterminal propeptide of procollagen type III, and hyaluronan in sera and tissue of patients with capsular contracture after augmentation with Trilucent breast implants. Plast Reconstr Surg. 2004;114:229-36.

32. Ulrich D, Ulrich F, Pallua N, Eisenmann-Klein M. Effect of tissue inhibitors of metalloproteinases and matrix metalloproteinases on capsular formation around smooth and textured silicone gel implants. Aesthetic Plast Surg. 2009;33:555-62.

33. Tan KT, Wijeratne D, Shih B, Baildam AD, Bayat A. Tumour necrosis factor-alpha expression is associated with increased severity of periprosthetic breast capsular contracture. Eur Surg Res. 2010;45:327-32.

34. Rosato RM, Dowden RV. Radiation therapy as a cause of capsular contracture. Ann Plast Surg. 1994;32:342-5

35. Katzel EB, Koltz PF, Tierney R, Williams JP, Awad HA, O’Keefe R J, et al. The impact of Smad3 loss of function on TGF-beta signaling and radiation-induced capsular contracture. Plast Reconstr Surg. 2011;127:2263-9.

36. Katzel EB, Koltz PF, Tierney R, Williams JP, Awad HA, O'Keefe RJ, et al. A novel animal model for studying silicone gel-related capsular contracture. Plast Reconstr Surg. 2010;126:1483-91.

37. Lipa JE, Qiu W, Huang N, Alman BA, Pang CY. Pathogenesis of radiation-induced capsular contracture in tissue expander and implant breast reconstruction. Plast Reconstr Surg. 2010;125:437-45

38. Whitfield GA, Horan G, Irwin MS, Malata CM, Wishart GC, Wilson CB Incidence of severe capsular contracture following implant-based immediate breast reconstruction with or without postoperative chest wall radiotherapy using 40 Gray in 15 fractions. Radiother Oncol. 2009;90:1417.

39. Macadam SA, Clugston PA, Germann ET. Retrospective case review of capsular contracture after two-stage breast reconstruction: is coloniza- tion of the tissue expander pocket associated with subsequent implant capsular contracture? Ann Plast Surg. 2004;53:420-4

40. Hvilsom GB, Holmich LR, Steding-Jessen M, Frederiksen K, Henriksen TF, Lipworth L, et al. Delayed breast implant reconstruction: Is radiation therapy associated with capsular contracture or reoperations? Ann Plast Surg. 2011;68:246-52.

41. Drucker-Zertuche M, Bargallo-Rocha E, Zamora-Del RR. Radiotherapy and immediate expander/implant breast reconstruction: should reconstruction be delayed? Breast J. 2011;17:365-70.

42. Pinsolle V, Grinfeder C, Mathoulin-Pelissier S, Faucher A. Complications analysis of 266 immediate breast reconstructions. J Plast Reconstr Aesthet Surg. 2006;59:1017-24.

43. Contant CM, van Geel AN, van der Holt B, Griep C, Tjong Joe Wai R, Wiggers T. Morbidity of immediate breast reconstruction (IBR) after mastectomy by a subpectorally placed silicone prosthesis: the adverse effect of radiotherapy. Eur J Surg Oncol. 2000;26:344-50.

44. Heneghan HM, Prichard RS, Lyons R, Regan PJ, Kelly JL, Malone C, et al. Quality of life after immediate breast reconstruction and skin-sparing mastectomy - a comparison with patients undergoing breast conserving surgery. Eur J Surg Oncol. 2011;37:937-43.

45. Behranwala KA, Dua RS, Ross GM, Ward A, A'Hern R, Gui GP. The influence of radiotherapy on capsule formation and aesthetic outcome after immediate breast reconstruction using biodimensional anatomical expander implants. J Plast Reconstr Aesthet Surg. 2006;59:1043-51.

46. Benediktsson K, Perbeck L. Capsular contracture around saline-filled and textured subcutaneously-placed implants in irradiated and non-irradiated breast cancer patients: five years of monitoring of a prospective trial. J Plast Reconstr Aesthet Surg. 2006;59:27-34.

47. Dancey A, Nassimizadeh A, Levick P. Capsular contracture - What are the risk factors? - A 14 year series of 1400 consecutive augmentations. J Plast Reconstr Aesthet Surg. 2012;65:213-8.

48. Vardanian AJ, Clayton JL, Roostaeian J, Shirvanian V, Da Lio A, Lipa JE, et al. Comparison of implant-based immediate breast reconstruc tion with and without acellular dermal matrix. Plast Reconstr Surg. 2011;128:403e-10

49. JoAnna Nguyen T, Carey JN, Wong AK. Use of human acellular dermal matrix in implant- based breast reconstruction: evaluating the evidence. J Plast Reconstr Aesthet Surg. 2011;64:1553-61.

50. Jansen LA, Macadam SA. The use of AlloDerm in postmastectomy alloplastic breast reconstruction: part II. A cost analysis. Plast Reconstr Surg. 2011;127:2245-54.

51. Maxwell GP, Gabriel A. Use of the acellular dermal matrix in revisionary aesthetic breast surgery. Aesthetic Plast Surg. 2009;29:485-93.

52. Orenstein SB, Qiao Y, Kaur M, Klueh U, Kreutzer DL, Novitsky YW. Human monocyte activation by biologic and biodegradable meshes in vitro. Surg Endosc. 2010;24:805-11.

53. Stump A, Holton LH 3rd, Connor J, Harper JR, Slezak S, Silverman RP. The use of acellular dermal matrix to prevent capsule formation around implants in a primate model. Plast Reconstr Surg. 2009;124:82-91.

54. Basu CB, Leong M, Hicks MJ. Acellular cadaveric dermis decreases the inflammatory response in capsule formation in reconstructive breast surgery. Plast Reconstr Surg. 2010;126:1842-7.

55. El-Sheikh Y, Tutino R, Knight C, Farrokhyar F, Hynes N. Incidence of capsular contracture in silicone versus saline cosmetic augmentation mammoplasty: A meta-analysis. Can J Plast Surg. 2008;16:211-5.

56. Escudero FJ, Guarch R, Lozano JA. Reaccion tisular a las protesis mamarias. Contractura capsular periprotesica. An Sist Sanit Navar. 2005;28:S41-53.

57. Wong $\mathrm{CH}$, Samuel $\mathrm{M}$, Tan BK, Song C. Capsular contracture in subglandular breast augmentation with textured versus smooth breast implants: a systematic review. Plast Reconstr Surg. 2006;118:1224-36.

58. Minami E, Koh IH, Ferreira JC, Waitzberg AF, Chifferi V, Rosewick TF, et al. The composition and behavior of capsules around smooth and textured breast implants in pigs. Plast Reconstr Surg. 2006;118:874-84

59. Barnsley GP, Sigurdson LJ, Barnsley SE. Textured surface breast implants in the prevention of capsular contracture among breast augmentation patients: a meta-analysis of randomized controlled trials. Plast Reconstr Surg. 2006;117:2182-90.

60. Poeppl N, Schreml S, Lichtenegger F, Lenich A, Eisenmann-Klein M Prantl L. Does the surface structure of implants have an impact on the formation of a capsular contracture? Aesthetic Plast Surg. 2007;31:1339.

61. Barr S, Hill E, Bayat A. Current implant surface technology: an examination of their nanostructure and their influence on fibroblast alignment and biocompatibility. Eplasty. 2009;9:e22

62. Ashley FL. A new type of breast prosthesis. Preliminary report. Plast 
Reconstr Surg. 1970;45:421-4.

63. Vazquez G, Pellon A. Polyurethane-coated silicone gel breast implants used for 18 years. Aesthetic Plast Surg. 2007;31:330-6.

64. Handel N, Cordray T, Gutierrez J, Jensen JA. A long-term study of outcomes, complications, and patient satisfaction with breast implants. Plast Reconstr Surg. 2006;117:757-67.

65. Barone FE, Perry L, Keller T, Maxwell GP. The biomechanical and histopathologic effects of surface texturing with silicone and polyurethane in tissue implantation and expansion. Plast Reconstr Surg. 1992;90:77-86.

66. Batich C, Williams J, King R. Toxic hydrolysis product from a biodegradable foam implant. J Biomed Mater Res. 1989;23:S311-9.

67. Sinclair TM, Kerrigan CL, Buntic R. Biodegradation of the polyurethane foam covering of breast implants. Plast Reconstr Surg. 1993;92:100313.

68. Food and Drug Administration: Update: Study of TDA released from polyurethane foam-covered breast implants. Silver Spring: FDA; 1995.

69. Handel N, Gutierrez J. Long-term safety and efficacy of polyurethane foam-covered breast implants. Aesthetic Surg J. 2006;26:265-74.

70. Carlesimo B, Cigna E, Fino P, Rusciani A, Tariciotti F, Staccioli S. Antibiotic therapy of transaxillary augmentation mammoplasty. In Vivo. 2009;23:357-62.

71. Darouiche RO, Meade R, Mansouri MD, Netscher DT. In vivo efficacy of antimicrobe-impregnated saline-filled silicone implants. Plast Reconstr Surg. 2002;109:1352-7.

72. Khan UD. Breast augmentation, antibiotic prophylaxis, and infection: comparative analysis of 1,628 primary augmentation mammoplasties assessing the role and efficacy of antibiotics prophylaxis duration. Aesthetic Plast Surg. 2010;34:42-7.

73. Carrico TJ, Cohen IK. Capsular contracture and steroid-related complications after augmentation mammaplasty. A preliminary study. Plast Reconstr Surg. 1979;64:377-80.

74. Oneal RM, Argenta LC. Late side effects related to inflatable breast prostheses containing soluble steroids. Plast Reconstr Surg. 1982;69:641-5.

75. Morykwas MJ, Argenta LC, Oneal RM, Kaiser DG. The fate of soluble steroids within breast prostheses in humans. Ann Plast Surg. 1990;24:427-30.

76. Richters CD, Paauw NJ, Mayen I, van Bloois L, Metselaer JM, Storm $\mathrm{G}$, et al. Administration of prednisolone phosphate-liposomes reduces wound contraction in a rat partial-thickness wound model. Wound Repair Regen. 2006;14:602-7.

77. Moreira M, Fagundes DJ, de Jesus Simoes M, Taha MO, Perez LM, Bazotte RB. The effect of liposome-delivered prednisolone on collagen density, myofibroblasts, and fibrous capsule thickness around silicone breast implants in rats. Wound Repair Regen. 2010;18:417-25.

78. Gancedo M, Ruiz-Corro L, Salazar-Montes A, Rincon AR, ArmendarizBorunda J. Pirfenidone prevents capsular contracture after mammary implantation. Aesthetic Plast Surg. 2008;32:32-40.

79. Zimman OA, Toblli J, Stella I, Ferder M, Ferder L, Inserra F. The effects of angiotensin-converting-enzyme inhibitors on the fibrous envelope around mammary implants. Plast Reconstr Surg. 2007;120:2025-33.

80. Kang Y, Lee DA, Higginbotham EJ. In vitro evaluation of antiproliferative potential of calcium channel blockers in human Tenon's fibroblasts. Exp Eye Res. 1997;64:913-25

81. Benlier E, Unal Y, Usta U, Top H, Aygit AC. Effect of verapamil on reduction of peri-implant capsular thickness. Aesthetic Plast Surg. 2009:33:570-5.

82. Marques M, Brown SA, Cordeiro ND, Rodrigues-Pereira P, Cobrado $\mathrm{ML}$, Morales-Helguera A, et al. Effects of fibrin, thrombin, and blood on breast capsule formation in a preclinical model. Aesthetic Surg J. 2011;31:302-9.

83. Marques M, Brown SA, Cordeiro ND, Rodrigues-Pereira P, Cobrado ML, Morales-Helguera A, et al. Effects of coagulase-negative staphylococci and fibrin on breast capsule formation in a rabbit model. Aesthet Surg J. 2011;31:420-8

84. Nordentoft T, Romer J, Sorensen M. Sealing of gastrointestinal anastomoses with a fibrin glue-coated collagen patch: a safety study. J Invest Surg. 2007;20:363-9.

85. Whitlock EL, Kasukurthi R, Yan Y, Tung TH, Hunter DA, Mackinnon SE. Fibrin glue mitigates the learning curve of microneurosurgical repair. Microsurgery. 2010;30:218-22.

86. Richards PJ, Turner AS, Gisler SM, Kraft S, Nuss K, Mark S, et al. Reduction in postlaminectomy epidural adhesions in sheep using a fibrin sealant-based medicated adhesion barrier. J Biomed Mater Res B Appl Biomater. 2010;92:439-46.

87. Farid M, Pirnazar JR. Pterygium recurrence after excision with conjunctival autograft: a comparison of fibrin tissue adhesive to absorbable su- tures. Cornea. 2009:28:43-5.

88. Osborne SF, Eidsness RB, Carroll SC, Rosser PM. The use of fibrin tissue glue in the repair of cicatricial ectropion of the lower eyelid. Ophthalmic Plast Reconstr Surg. 2010;26:409-12.

89. Kavanagh MC, Ohr MP, Czyz CN, Cahill KV, Perry JD, Holck DE, et al. Comparison of fibrin sealant versus suture for wound closure in Muller muscle-conjunctiva resection ptosis repair. Ophthalmic Plast Reconstr Surg. 2009;25:99-102.

90. Biedner B, Rosenthal G. Conjunctival closure in strabismus surgery: Vicryl versus fibrin glue. Ophthalmic Surg lasers. 1996;27:967.

91. Chan SM, Boisjoly H. Advances in the use of adhesives in ophthalmology. Curr Opin Ophthalmol. 2004;15:305-10.

92. Sarnicola V, Vannozzi L, Motolese PA. Recurrence rate using fibrin glueassisted ipsilateral conjunctival autograft in pterygium surgery: 2-year follow-up. Cornea. 2010;29:1211-4.

93. Saed GM, Kruger M, Diamond MP. Expression of transforming growth factor-beta and extracellular matrix by human peritoneal mesothelial cells and by fibroblasts from normal peritoneum and adhesions: effect of Tisseel. Wound Repair Regen. 2004;12:557-64.

94. Ruiz-de-Erenchun R, Dotor de las Herrerias J, Hontanilla B. Use of the transforming growth factor-beta1 inhibitor peptide in periprosthetic capsular fibrosis: experimental model with tetraglycerol dipalmitate. Plast Reconstr Surg. 2005:116:1370-8.

95. Diao ZY, Fu HL, Nie CL, Hao LJ, Yang DP, Chen WH. Controlled release of transforming growth factor-beta receptor kinase inhibitor from thermosensitive Chitosan-based hydrogel: application for prevention of capsular contracture. Chin Med J. 2011;124:284-90.

96. Tumerdem-Ulug B, Kuran I, Ozden BC, Mete O, Kemikler G, Aktas S, et al. Does hyperbaric oxygen administration before or after irradiation decrease side effects of irradiation on implant sites? Ann Plast Surg. 2011;67:62-7.

97. Mendes FH, Viterbo F, DeLucca L. The influence of external ultrasound on the histologic architecture of the organic capsule around smooth silicone implants: experimental study in rats. Aesthetic Plast Surg. 2008;32:442-50

98. Cardenas-Camarena L, Paillet JC, Briseno R. Electrostimulation: uses and applications for periprosthetic capsular contracture: experimental model. Aesthetic Plast Surg. 2005;29:410-4

99. Ajmal N, Riordan CL, Cardwell N, Nanney LB, Shack RB. The effectiveness of sodium 2-mercaptoethane sulfonate (mesna) in reducing capsular formation around implants in a rabbit model. Plast Reconstr Surg. 2003;112:1455-61.

100. M DN, Cobanoglu U, Ambarcioglu O, Topal U, Kutlu N. Effect of amniotic fluid on peri-implant capsular formation. Aesthetic Plast Surg. 2005;29:174-80.

101. Camirand A, Doucet J. Breast augmentation: teaching our patients how compression can help prevent capsular contracture. Aesthetic Plast Surg. 2000;24:221-6.

102. Collis N, Sharpe DT. Recurrence of subglandular breast implant capsular contracture: anterior versus total capsulectomy. Plast Reconstr Surg. 2000;106:792-7.

103. Spear SL, Carter ME, Ganz JC. The correction of capsular contracture by conversion to 'dual-plane' positioning: technique and outcomes. Plast Reconstr Surg. 2006;118:103S-13.

104. Siggelkow W, Lebrecht A, Kolbl H, Faridi A. Dual-plane implant positioning for capsular contracture of the breast in combination with mastopexy. Arch Gynecol Obstet. 2005;273:79-85.

105. Baran CN, Peker F, Ortak T, Sensoz O, Baran NK. A different strategy in the surgical treatment of capsular contracture: leave capsule intact. Aesthetic Plast Surg. 2001;25:427-31.

106. Xue H, Lee SY. Correction of capsular contracture by insertion of a breast prosthesis anterior to the original capsule and preservation of the contracted capsule: technique and outcomes. Aesthetic Plast Surg. 2011;35:1056-60

107. Schlesinger SL, Ellenbogen R, Desvigne MN, Svehlak S, Heck R. Zafirlukast (Accolate): A new treatment for capsular contracture. Aesthetic Plast Surg. 2002:22:329-36.

108. Bastos EM, Sabino Neto M, Garcia EB, Veiga DF, Han YA, Denadai R, et al. Effect of zafirlukast on capsular contracture around silicone implants in rats. Acta Cir Bras. 2012;27:1-6.

109. Spano A, Palmieri B, Taidelli TP, Nava MB. Reduction of capsular thickness around silicone breast implants by zafirlukast in rats. Eur Surg Res. 2008:41:8-14

110. Bastos EM, Neto MS, Alves MT, Garcia EB, Santos RA, Heink T, et al. Histologic analysis of zafirlukast's effect on capsule formation around silicone implants. Aesthetic Plastic Surg. 2007:31:559-65. 
111. Reid RR, Greve SD, Casas LA. The effect of zafirlukast (Accolate) on early capsular contracture in the primary augmentation patient: a pilot study. Aesthetic Plast Surg. 2005;25:26-30.

112. Scuderi N, Mazzocchi M, Rubino C. Effects of zafirlukast on capsular contracture: controlled study measuring the mammary compliance. Int $\mathrm{J}$ Immunopathol Pharmacol. 2007;20:577-84.

113. Huang CK, Handel N. Effects of Singulair (montelukast) treatment for capsular contracture. Aesthetic Plast Surg. 2010;30:404-8.

114. Gryskiewicz JM. Investigation of accolate and singulair for treatment of capsular contracture yields safety concerns. Aesthetic Plast Surg. 2003;23:98-101.

115. Danese S, De Vitis I, Gasbarrini A. Severe liver injury associated with zafirlukast. Ann Intern Med. 2001;135:930.

116. Physicians' desk reference. Singulair tablets and chewable tablets (montelukast sodium). Montvale: Medical Economics; 2002.
117. Goldstein M, Anoia J, Black M. Montelukast-induced hepatitis. Ann Intern Med. 2004;140:586-7.

118. Veras-Castillo ER, Cardenas-Camarena L, Lyra-Gonzalez I, MunozValle JF, Lucano-Landeros S, Guerrerosantos J, et al. Controlled clinical trial with pirfenidone in the treatment of breast capsular contracture: Association of TGF-beta polymorphisms. Ann Plast Surg. 2011.

119. Sconfienza LM, Murolo C, Callegari S, Calabrese M, Savarino E, Santi $P$, et al. Ultrasound-guided percutaneous injection of triamcinolone acetonide for treating capsular contracture in patients with augmented and reconstructed breast. Eur Radiol. 2011;21:575-81.

120. Le Louarn C, Buis J, Auclair E. Flector tissugel used to treat capsular contracture after breast augmentation surgery. Aesthetic Plast Surg. 2008;32:453-8

121.Planas J, Cervelli V, Planas G. Five-year experience on ultrasonic treatment of breast contractures. Aesthetic Plast Surg. 2001;25:89-93. 


\section{Etiopatogenia e Tratamento da Contractura Capsular Mamária Acta Med Port 2013:26:737-745}

Publicado pela Acta Médica Portuguesa, a Revista Científica da Ordem dos Médicos

Av. Almirante Gago Coutinho, 151 1749-084 Lisboa, Portugal.

Tel: +351218428 215

E-mail: submissao@actamedicaportuguesa.com

www.actamedicaportuguesa.com

ISSN:0870-399X | e-ISSN: 1646-0758

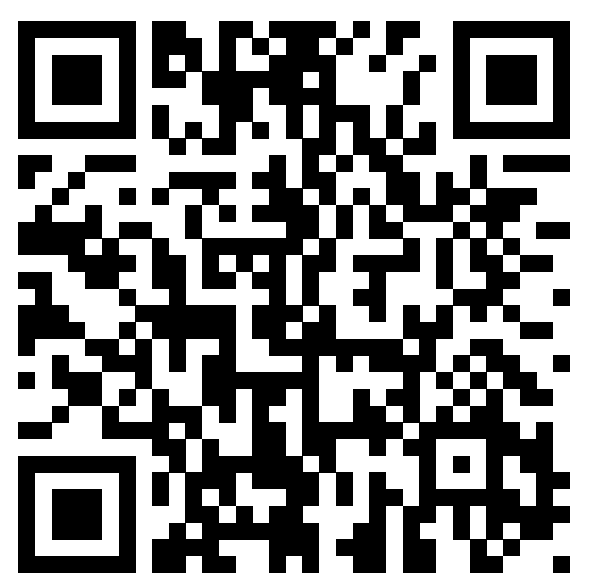

